\title{
Inverse seesaw mechanism and portal dark matter
}

\author{
Chakrit Pongkitivanichkul, ${ }^{1,3, *}$ Nakorn Thongyoi, ${ }^{1, \dagger}$ and Patipan Uttayarat $\odot^{2,3, *}$ \\ ${ }^{1}$ Department of Physics, Khon Kaen University, 123 Mitraphap Rd., Khon Kaen 40002, Thailand \\ ${ }^{2}$ Department of Physics, Srinakharinwirot University, 114 Sukhumvit 23rd Rd., \\ Wattana, Bangkok 10110, Thailand \\ ${ }^{3}$ National Astronomical Research Institute of Thailand, Chiang Mai 50180, Thailand
}

(Received 9 June 2019; published 28 August 2019)

\begin{abstract}
We study the phenomenology of the inverse seesaw mechanism in the scalar-Higgs portal dark matter model. The model is an extension of the Standard Model including two additional neutrinos, a singlet scalar and a fermionic dark matter. We consider the inverse seesaw mechanism in which the mass of two additional neutrinos are made dynamic by the singlet scalar. We found that the natural scale for the scalar vacuum expectation value is naturally close to the weak scale. Motivated by this fact, we focus on the possibility of the singlet scalar connecting with dark matter; i.e., the scalar is also the mediator between dark sector and the Standard Model. We perform a numerical analysis over the parameter space subject to the indirect and direct detection constraints. The feasible region of the parameter space will be discussed.
\end{abstract}

DOI: $10.1103 /$ PhysRevD.100.035034

\section{INTRODUCTION}

The discovery of the Higgs boson in 2012, together with decades of electroweak precision tests, has been hailed as the remarkable success of the Standard Model (SM) of particle physics. However, the existence of neutrino masses and dark matter (DM) strongly suggests an extension beyond the Standard Model that requires new degrees of freedom. The connection between these new physics is therefore simplistic yet tantalizing.

Seesaw mechanisms are considered the best explanation for the smallness of the neutrino mass. In the minimal realization of the seesaw mechanism, a right-handed neutrino is introduced to SM, in which the active lefthanded neutrino gains its mass from its mixing with the right-handed neutrino. The mass can be obtained from the formula $m_{\nu}=\frac{m_{D}^{2}}{M}$, where $m_{D}$ is the Dirac mass and $M$ is the Majorana mass of the right-handed neutrino. In the seesaw mechanism, the sub-electron volt neutrino mass requires $M \sim 10^{16} \mathrm{GeV}$. This huge difference between the electroweak scale and the seesaw scale leads to a strong suppression for any potential phenomenological signals from accelerator experiments and astronomical

\footnotetext{
*chakpo@kku.ac.th

†nakorn_t@kkumail.com

"patipan@g.swu.ac.th
}

Published by the American Physical Society under the terms of the Creative Commons Attribution 4.0 International license. Further distribution of this work must maintain attribution to the author(s) and the published article's title, journal citation, and DOI. Funded by SCOAP ${ }^{3}$. observations. Although the minimal seesaw mechanism provides an interesting explanation for neutrino mass, it certainly lacks the testability and thus diminishes the chance for connecting the origin of neutrino mass and DM observables.

To bring the seesaw scale closer to the electroweak scale, one can employ the so-called inverse seesaw mechanism. Inspired by string/M theory, the SM is extended by two sterile neutrinos and an electroweak scalar singlet [1]. It has been shown that the small neutrino mass can be generated from new physics around the tera-electron-volt scale. The connection with DM within the inverse seesaw context has recently gained interest [2-6]. It is well known that DM cannot take part directly in the seesaw mechanism [7-11]. If the sterile neutrino is the DM, it would decay into gamma rays and active neutrino. On the other hand, the option with the singlet scalar being DM is also limited due to its mixing with the Higgs, which leads to the shorter lifetime. To avoid such pitfalls, one can instead utilize the heavy neutrinos or the singlet scalar field as a mediator to connect with the dark sector $[6,12]$.

In this paper, we are interested in exploring the possibilities of connecting DM to the inverse seesaw model. In particular, we will consider the model in which the Dirac mass for the additional sterile neutrinos $M_{D}$ is explained by the dynamic of a scalar field mediator. The mediator is then connected to the fermionic dark sector via scalar and pseudoscalar coupling. The paper is organized as follows. First, we provide the setup of the model in Sec. II. In this section, the inverse seesaw mechanism in which the lightest neutrino is identified with the SM active neutrino is described. The neutrino couplings are derived, and the 
TABLE I. The field contents and their transformation properties.

\begin{tabular}{lccc}
\hline \hline & $S U(2)_{L}$ & $U(1)_{Y}$ & $Z_{2}$ \\
\hline$L$ & 2 & $-1 / 2$ & +1 \\
$H$ & 2 & $1 / 2$ & +1 \\
$N_{1}$ & 1 & 0 & +1 \\
$N_{2}$ & 1 & 0 & -1 \\
$\Phi$ & 1 & 0 & -1 \\
$\chi$ & 1 & 0 & -1 \\
\hline \hline
\end{tabular}

scalar mediator mixing with Higgs is investigated. Phenomenology and the constraints on the model are presented in Sec. III. The invisible $Z$ boson decay is discussed. The indirect detection via the gamma-ray and neutrino telescope is investigated. Then, the direct detection via nucleon scattering is studied. The scan of all parameter space of the model subject to constraints is shown in Sec. IV. We finally conclude in Sec. V.

\section{MODEL}

To construct the model with the inverse seesaw mechanism, we extend the SM by adding two additional fermions: the right-handed $N_{1}$ and the left-handed $N_{2}$. We also add an electroweak singlet scalar $\Phi$ of which the vacuum expectation value (vev) is responsible for the Dirac mass for the new fermions. In addition to the SM gauge groups, the $Z_{2}$ discrete symmetry in which all the SM fields are even is imposed, in order to obtain the neutrino mass matrix texture desired by the model; see Table I.

In a four-component notation, the Lagrangians for the neutrino sector and the scalar sector are

$$
\begin{gathered}
\mathcal{L}_{\mathrm{N}}=-y \bar{L} \tilde{H} N_{1 \mathrm{R}}-g \Phi \overline{N_{2 \mathrm{~L}}} N_{1 \mathrm{R}} \\
-\frac{\mu_{N}}{2}\left(\overline{N_{1 R}^{c}} N_{1 R}+\overline{N_{2 L}^{c}} N_{2 L}\right)+\text { H.c. }, \\
\mathcal{L}_{\text {scalar }}=\left(D_{\mu} H\right)^{\dagger}\left(D^{\mu} H\right)+\frac{1}{2}\left(\partial_{\mu} \Phi\right)\left(\partial^{\mu} \Phi\right)-V(H, \Phi),
\end{gathered}
$$

where

$$
\begin{aligned}
V(H, \Phi)= & -\mu^{2} H^{\dagger} H+\lambda\left(H^{\dagger} H\right)^{2}-\frac{\mu_{\phi}^{2}}{2} \Phi^{2}+\frac{\lambda_{\phi}}{4} \Phi^{4} \\
& +\frac{\lambda_{\phi H}}{2} \Phi^{2} H^{\dagger} H .
\end{aligned}
$$

In the above equations, we have $\tilde{H}=i \sigma_{2} H^{*}$ and $\psi^{c}=C \bar{\psi}^{T}$, and $\mu_{N}$ is Majorana mass of the heavy neutrino. Note the $\mu_{N}$ term would violate the U(1) lepton number symmetry. Thus, we expect it to be small. Moreover, the smallness of $\mu_{N}$ is technically natural by 't Hooft's naturalness principle. Lastly, we can extend our model such that small $\mu_{N}$ is generated in a fashion similar to models considered in Refs. [2,13]. This model is quite different than the recent study of Ref. [6], in which the dynamic part of the inverse seesaw is in the Majorona term.

The potential in Eq. (3) admits nontrivial vevs for the two scalar fields. We can expand both $H$ and $\Phi$ around their vevs as

$$
H=\frac{1}{\sqrt{2}}\left(\begin{array}{c}
w^{+} \\
h^{\prime}+i z+v
\end{array}\right), \quad \Phi=\phi^{\prime}+v_{\phi},
$$

where $w^{+}$and $z$ are the would-be Goldstone bosons eaten by the $W^{+}$and $Z$ gauge bosons. Thus, we see that the scalar sector contains two real degrees of freedom, denoted by $h^{\prime}$ and $\phi^{\prime}$.

Because of the scalar mixing with the Higgs, massive $N_{1}, N_{2}$, and $\phi^{\prime}$ are not stable and hence cannot be a good DM candidate. We assume that DM resides in a separated sector that is connected to our sector by the scalar $\Phi$. We will further assume for simplicity that DM is a fermion. Thus, the Lagrangian for DM is given by

$$
\mathcal{L}_{\mathrm{DM}}=\Phi \bar{\chi}\left(G+i \tilde{G} \gamma^{5}\right) \chi+M \bar{\chi} \chi,
$$

where $G$ is a coupling and $\tilde{G}$ is a pseudoscalar coupling. Note that this dark sector explicitly breaks the $Z_{2}$ symmetry and contains the $C P$ violation, which will be mediated to the neutrino sector.

\section{A. Neutrino mass}

Neutrino masses arise from the Yukawa interactions in Eq. (1),

$$
\begin{aligned}
\mathcal{L}_{\mathrm{N}} \supset & -\frac{1}{2 \sqrt{2}} y v\left(\overline{\nu_{L}} N_{1 \mathrm{R}}+\overline{N_{1 \mathrm{R}}^{\mathrm{c}}} \nu_{\mathrm{L}}^{c}\right) \\
& -\frac{1}{2} g v_{\phi}\left(\overline{N_{2 \mathrm{~L}}} N_{1 \mathrm{R}}+\overline{N_{1 \mathrm{R}}^{c}} N_{2 \mathrm{~L}}^{c}\right) \\
& -\frac{\mu_{N}}{2}\left(\overline{N_{1 R}^{c}} N_{1 R}+\overline{N_{2 L}^{c}} N_{2 L}\right)+\text { H.c. },
\end{aligned}
$$

where we used the fact that $\overline{\nu_{\mathrm{L}}} N_{1 \mathrm{R}}=\overline{N_{1 \mathrm{R}}^{c}} \nu_{\mathrm{L}}^{c}$. The Lagrangian for the neutrino sector becomes the mass matrix under the basis $\psi_{R}=\left(\nu_{\mathrm{L}}^{c}, N_{1}, N_{2}^{c}\right)$,

$$
\mathcal{L}_{\mathrm{N}}^{\mathrm{Mass}}=-\frac{1}{2} \overline{\psi_{R}^{c}} M \psi_{R},
$$

where

$$
M=\left(\begin{array}{ccc}
0 & y v / \sqrt{2} & 0 \\
y v / \sqrt{2} & \mu_{N} & g v_{\phi} \\
0 & g v_{\phi} & \mu_{N}
\end{array}\right) .
$$

We can diagonalize the mass matrix by an $S O(3)$ rotation matrix $R$, 


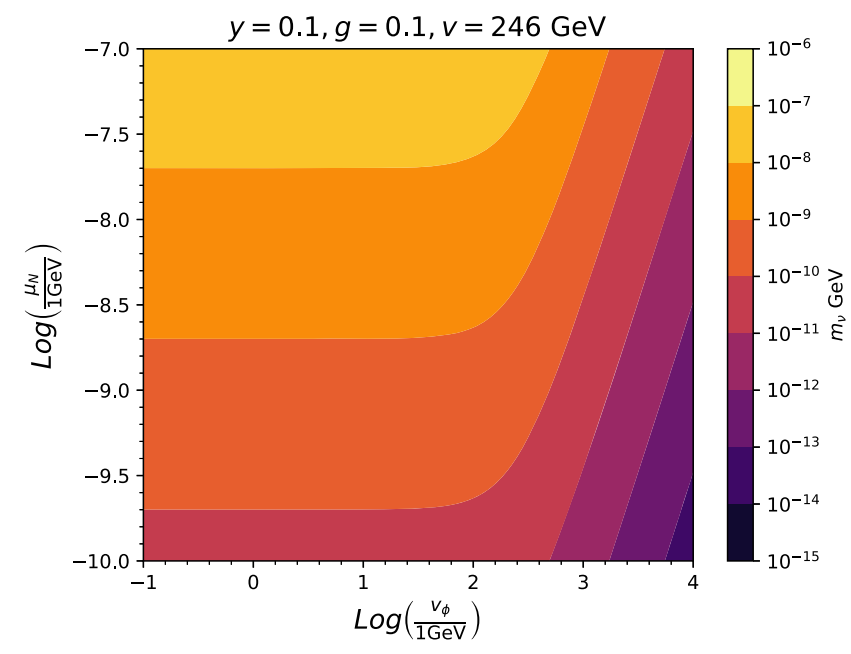

FIG. 1. The contour plot showing the value of $\log _{10}\left(\frac{m_{\nu}}{1 \mathrm{GeV}}\right)$.

$$
\left(\begin{array}{c}
\nu_{l}^{c} \\
N_{1 R} \\
N_{2 L}^{c}
\end{array}\right) \equiv R\left(\begin{array}{c}
\psi_{1 R} \\
\psi_{2 R} \\
\psi_{3 R}
\end{array}\right) \equiv R \Psi_{R}
$$

where $\psi_{i R}$ 's are the mass eigenstates. Without loss of generality, we take $m_{\psi_{1}}<m_{\psi_{2}}<m_{\psi_{3}}$. That is, $\psi_{1 R}$ is the observed light neutrino. To the lowest nontrivial order in $\mu_{N}$, we have

$$
\begin{aligned}
& M_{D}=\operatorname{diag}\left(\frac{y^{2} v^{2}}{y^{2} v^{2}+2 g^{2} v_{\phi}^{2}} \mu_{N},\right. \\
& \left.\frac{1}{\sqrt{2}} \sqrt{y^{2} v^{2}+2 g^{2} v_{\phi}^{2}} \mp \frac{1}{2} \frac{y^{2} v^{2}+4 g^{2} v_{\phi}^{2}}{y^{2} v^{2}+2 g^{2} v_{\phi}^{2}} \mu_{N}\right)+\mathcal{O}\left(\mu_{N}^{2}\right) .
\end{aligned}
$$

Notice in the limit $\mu_{N} \rightarrow 0, m_{\psi_{1}} \rightarrow 0$ and $m_{\psi_{2}}=m_{\psi_{3}}$. As an illustrative example, the smallest eigenvalue in the case $y=g=0.1$ is shown in Fig. 1 .

\section{B. Neutrino couplings}

First, we consider the neutrino Yukawa couplings. In the interaction basis, we have

$$
\begin{aligned}
\mathcal{L}_{Y} \supset & -\frac{y}{\sqrt{2}} h^{\prime} \overline{\overline{\nu_{L}}} N_{1 R}-g \phi^{\prime} \overline{N_{2 L}} N_{1 R}+\text { H.c. } \\
= & -\frac{y}{2 \sqrt{2}} h^{\prime}\left(\overline{\overline{\nu_{L}}} N_{1 R}+\overline{\nu_{L}^{c}} N_{1 R}^{c}\right) \\
& -\frac{g}{2} \phi^{\prime}\left(\overline{N_{2 L}} N_{1 R}+\overline{N_{2 L}^{c}} N_{1 R}^{c}\right)+\text { H.c. } \\
= & -\frac{y}{2 \sqrt{2}} h^{\prime} R_{1 j} R_{2 k}\left(\overline{\Psi_{R}^{c}}+\overline{\Psi_{R}}\right)_{j}\left(\Psi_{R}+\Psi_{R}^{c}\right)_{k} \\
& -\frac{g}{2} \phi^{\prime} R_{2 k} R_{3 j}\left(\overline{\Psi_{R}^{c}}+\overline{\Psi_{R}}\right)_{j}\left(\Psi_{R}+\Psi_{R}^{c}\right)_{k}+\text { H.c. }
\end{aligned}
$$

Introducing Majorana field $\Psi=\Psi_{R}+\Psi_{R}^{c}$, the above interaction can be written as

$$
\mathcal{L}_{Y}=-\frac{1}{2}\left[\frac{y}{\sqrt{2}} h^{\prime} R_{1 j} R_{2 k}+g \phi^{\prime} R_{2 k} R_{3 j}\right] \bar{\Psi}_{j} \Psi_{k}+\text { H.c. }
$$

Note that in the above equation $h^{\prime}$ and $\phi^{\prime}$ are not in the mass basis. They can be rotated to the mass basis by an orthogonal rotation; see Eq. (16).

Now, we consider the couplings of the neutrino with gauge bosons. They arise from the kinetic term of the lepton doublet, $\ell$,

$$
\begin{aligned}
i \bar{\ell} \not \ell & \supset i \bar{\ell}\left(-i \frac{e}{\sqrt{2} s_{\theta_{W}}}\left(W^{+} \sigma^{+}+W^{-} \sigma^{-}\right)\right. \\
& \left.-i \frac{e}{2 s_{\theta_{W}} c_{\theta_{W}}} Z\left(c_{\theta_{W}}^{2} \sigma^{3}+s_{\theta_{W}}^{2}\right)\right) e \\
& \supset \frac{e}{2 s_{\theta_{W}}}\left[\sqrt{2}\left(\overline{e_{L}} W^{-} \nu_{L}+\overline{\nu_{L}} W^{+} e_{L}\right)+\frac{1}{c_{\theta_{W}}} \overline{\nu_{L}} \not L \nu_{L}\right],
\end{aligned}
$$

where $c_{\theta_{W}}\left(s_{\theta_{W}}\right)$ is the cosine (sine) of the Weinberg angle, $\sigma^{ \pm}=\left(\sigma^{1} \pm i \sigma^{2}\right) / 2$, and $\sigma^{i}$ s are the Pauli matrices. In terms of the physical basis, we have

$$
\begin{aligned}
\mathcal{L}_{\text {gauge }} \supset & \frac{e}{\sqrt{2} s_{\theta_{W}}} R_{1 j}\left(\overline{e_{L}} W^{-} P_{L} \Psi_{j}+\overline{\Psi_{j}} W^{+} P_{L} e_{L}\right) \\
& +\frac{1}{2} \frac{e}{c_{\theta_{w}} s_{\theta_{w}}} R_{1 j} R_{1 k} \overline{\Psi_{k}} \not P_{L} \Psi_{j} .
\end{aligned}
$$

\section{Scalar mixing}

Because of the scalar potential in Eq. (3), the field $h^{\prime}$ and $\phi^{\prime}$ are allowed to mix. The mass matrix, in the basis $\left(h^{\prime}, \phi^{\prime}\right)$, is

$$
M^{2}=\left(\begin{array}{cc}
\lambda v^{2} & \lambda_{\phi H} v v_{\phi} \\
\lambda_{\phi H} v v_{\phi} & \lambda_{\phi} v_{\phi}^{2}
\end{array}\right) .
$$

We can diagonalize the mass matrix by an orthogonal rotation to the physical basis,

$$
\left(\begin{array}{l}
h \\
\phi
\end{array}\right)=\left(\begin{array}{cc}
\cos \theta & -\sin \theta \\
\sin \theta & \cos \theta
\end{array}\right)\left(\begin{array}{l}
h^{\prime} \\
\phi^{\prime}
\end{array}\right)
$$

where the mixing angle is determined by

$$
\tan 2 \theta=\frac{\lambda_{\phi H} v v_{\phi}}{\lambda_{\phi} v_{\phi}^{2}-\lambda v^{2}}
$$

The masses of the two physical states are 


$$
m_{h, \phi}^{2}=\lambda v^{2}+\lambda_{\phi} v_{\phi}^{2} \mp \sqrt{\left(\lambda_{\phi} v_{\phi}^{2}-\lambda v^{2}\right)^{2}+\lambda_{\phi H}^{2} v^{2} v_{\phi}^{2}} .
$$

The mixing angle $\theta$ is constrained by the LHC $125 \mathrm{GeV}$ Higgs measurements. So far, the measurements have been consistent with the SM predictions [14-16]. Thus, we expect the mixing angle $\theta$ to be small.

Finally, for later convenience, we give expressions for the parameters in the scalar potential in terms of physical masses, vevs, and the mixing angle:

$$
\begin{gathered}
\lambda=\frac{c_{\theta}^{2} m_{h}^{2}+s_{\theta}^{2} m_{\phi}^{2}}{v^{2}}, \\
\lambda_{\phi}=\frac{s_{\theta}^{2} m_{h}^{2}+c_{\theta}^{2} m_{\phi}^{2}}{v_{\phi}^{2}}, \\
\lambda_{\phi H}=\frac{c_{\theta} s_{\theta}\left(m_{\phi}^{2}-m_{h}^{2}\right)}{v v_{\phi}} .
\end{gathered}
$$

The scalar potential is bounded from below, provided $\lambda_{\phi H}>0$; therefore, we will strictly work in the case in which $m_{\phi}>m_{h}$.

\section{Dark matter}

The coupling of $\Phi$ with $\chi$ in Eq. (5) gives extra contributions to the DM mass, $m_{\chi}$. This can be seen by making a chiral rotation,

$$
\chi \rightarrow \chi^{\prime}=e^{i \alpha \gamma_{5}} \chi
$$

where $\alpha=\frac{1}{2} \tan ^{-1}\left(\frac{v_{\phi} \tilde{G}}{M+v_{\phi} G}\right)$. After chiral rotation, the mass of $\chi$ is

$$
m_{\chi}=\sqrt{\frac{\left(\sqrt{2} M+v_{\phi} G\right)^{2}+\left(v_{\phi} \tilde{G}\right)^{2}}{2}} .
$$

The interaction Lagrangian becomes

$$
\mathcal{L}=\frac{v_{\phi}\left(G^{2}+\tilde{G}^{2}\right)}{m_{\chi}} \phi \overline{\chi^{\prime}} \chi^{\prime}+\frac{M}{m_{\chi}} \phi \overline{\chi^{\prime}}\left(G+i \tilde{G} \gamma_{5}\right) \chi^{\prime} .
$$

Notice that if $M=0$ the interaction Lagrangian would contain no pseudoscalar coupling after the chiral transformation. Note also in the limit $v_{\phi}=0$, the chiral rotation leaves the Lagrangian unchanged.

\section{PHENOMENOLOGY}

\section{A. Invisible decay of $Z$}

The mixing between the neutrinos impacts the coupling of light neutrinos to the $Z$ boson; see Eq. (14). This results in a modification to the partial decay width of the $Z$ boson into neutrinos. The invisible decay width of the $Z$ boson has been measured very precisely, $\Gamma_{\text {inv }}^{\exp }=499.0 \pm 1.5 \mathrm{MeV}$, while the $\mathrm{SM}$ prediction is $\Gamma_{\text {inv }}^{\mathrm{SM}}=501.66 \pm 0.05 \mathrm{MeV}$ [17]. If we assume only $Z \rightarrow \psi_{1} \psi_{1}$ is kinematically allowed, we would get

$$
\Gamma_{\mathrm{inv}}=R_{11}^{4} \Gamma_{\mathrm{inv}}^{\mathrm{SM}},
$$

where $R_{11}$ is the 1-1 component of the rotation matrix defined in Eq. (9). This places a $2 \sigma$ limit on $R_{11}$ as

$$
R_{11}^{4} \gtrsim \frac{496}{501} \Rightarrow R_{11} \gtrsim 0.997 .
$$

It translates, in terms of Lagrangian parameters, to the bound

$$
\frac{v_{\phi}}{\sqrt{\left(\frac{y}{\sqrt{2} g}\right)^{2} v^{2}+v_{\phi}^{2}}} \gtrsim 0.997 .
$$

To get a feel for this constraint, let us take $v=246 \mathrm{GeV}$ and $v_{\phi}=1000 \mathrm{GeV}$; we get $y / g \lesssim 0.43$.

\section{B. $125 \mathrm{GeV}$ Higgs data}

The mixing angle $\theta$ changes the coupling of the $125 \mathrm{GeV}$ Higgs boson to other SM particles. These couplings have been measured to about $10 \%$ accuracy at the LHC. All the measurements can be parametrized in terms of a coupling strength modifier,

$$
\mu_{i}^{f}=\frac{\sigma_{i}}{\sigma_{i}^{(\mathrm{SM})}} \frac{\mathrm{Br}^{f}}{\mathrm{Br}_{(\mathrm{SM})}^{f}},
$$

where $i$ indicates the production cross section channel of the 125 Higgs boson and $f$ indicates the branching ratio channel. Using both LHC Run 1 [14] and Run 2 $[15,16]$ data, we deduce the overall best-fit value for $\mu=1.09 \pm 0.07$.

In our model, all the Higgs measurements are modified by the mixing angle, $\cos \theta$. Thus, the predicted value of $\mu$ is $\hat{\mu}=\cos ^{4} \theta$. Therefore, consistency with the Higgs data requires $|\cos \theta| \geq 0.9931$ at $95 \%$ confidence levels.

\section{Indirect detection}

DM $\chi$ can self-annihilate through its interaction with $\Phi$; see Eq. (5). We give explicit expressions for all the 2-2 annihilation channels of $\chi$ in Appendix A. From there, we see that the annihilations into the SM gauge bosons and fermions are suppressed by the scalar mixing angle. Thus, the main annihilation channels for $\chi$ are $\chi \bar{\chi} \rightarrow \phi \phi, \phi h, h h$ and $\chi \bar{\chi} \rightarrow \psi \gamma \bar{\psi}$. The annihilation into a neutrino can be looked for at neutrino telescopes such as IceCUBE [18]. For annihilations into $h$ and $\phi$, they can subsequently 
decays into photons, which can be looked for with gammaray telescopes such as Fermi-LAT [19].

\section{Gamma ray}

The gamma-ray flux produced from DM annihilation is given by

$$
\frac{d \Phi}{d E_{\gamma}}=\frac{J R_{\mathrm{sc}} \rho_{\mathrm{sc}}^{2}}{8 \pi m_{\mathrm{dm}}^{2}} \sum_{i, j}\langle\sigma v\rangle_{i} \mathrm{Br}_{i j} \frac{d N_{j}}{d E_{\gamma}}
$$

where $i$ runs over different scalar annihilation channels, $j$ runs over different $\mathrm{SM}$ final states, $\mathrm{Br}_{i j}$ is the branching ratio from the initial state $i$ into the SM final state $j$, and $\frac{d N_{j}}{d E_{\gamma}}$ gives the gamma-ray spectrum from the SM particle $j$. $R_{\mathrm{sc}}=8.5 \mathrm{kpc}$ and $\rho_{\mathrm{sc}}$ are normalization constants introduced to make $J$ dimensionless. Here, $R_{\mathrm{sc}}$ is the distance between the Sun and Milky Way's center, $\rho_{\text {sc }}$ is the DM density at position of the Sun. The $J$ factor is the typical average line of sight integral over the DM halo,

$$
J=\frac{1}{2 R_{\mathrm{sc}} \rho_{\mathrm{sc}}^{2}} \int_{-1}^{1} d \cos \theta \int_{0}^{l_{\max }} d l \rho^{2}(x)
$$

where $x=\sqrt{R_{\mathrm{sc}}^{2}-2 l R_{\mathrm{sc}} \cos \theta+l^{2}}$ is the distance from the Galactic Center to the position along the line of sight and $l_{\text {max }}=\sqrt{R_{\text {halo }}^{2}-R_{\mathrm{sc}}^{2} \sin ^{2} \theta}+R_{\mathrm{sc}} \cos \theta$ is the distance along the line of sight to the edge of the Galaxy. The integral over the line of sight gives the value of $J=3.34$ for the NavarroFrenk-White profile and $J=1.60$ for the Burkert profile. In our analysis, we use the Navarro-Frenk-White profile. One can easily translate our result to other DM profiles by an appropriate rescaling of the $J$ factor.

The gamma-ray spectrum coming from the chargedparticle final states can be obtained through computer simulations. Since we only need a ballpark estimation in order to obtain the constraint, the spectrum is assumed to have a power-law relation. However, in our case, the SM particles $j=b, t, u, W^{ \pm} / Z$ are produced from the subsequent decay of scalar particles $\chi \bar{\chi} \rightarrow \phi \phi, h h, \phi h \rightarrow$ $j \bar{j} j^{\prime} \bar{j}^{\prime}$. In the rest frame of the scalar $\phi$, (or $h$ ), the 4-momenta of the final-state particles are isotropically distributed. Boosting back to the DM center-of-mass frame, the energy of the final states $E_{j}$ ranges between

$$
\begin{aligned}
E_{\min }^{\phi, h} & =\frac{m_{\chi}}{2}\left(1-\sqrt{1-\left(\frac{m_{\phi, h}}{m_{\chi}}\right)^{2}} \sqrt{1-\left(\frac{2 m_{j}}{m_{\phi, h}}\right)^{2}}\right) \\
E_{\max }^{\phi, h} & =\frac{m_{\chi}}{2}\left(1+\sqrt{1-\left(\frac{m_{\phi, h}}{m_{\chi}}\right)^{2}} \sqrt{1-\left(\frac{2 m_{j}}{m_{\phi, h}}\right)^{2}}\right) .
\end{aligned}
$$

Averaging all possible directions, the differential probability of finding the SM particle with energy $E_{j}$ is

$$
\begin{aligned}
\left(\frac{d P}{d E_{j}}\right)_{\phi, h}= & \frac{4}{\pi m_{\chi}} \frac{1}{\left(1-\frac{m_{\phi, h}^{2}}{m_{\chi}^{2}}\right)\left(1-\frac{4 m_{j}^{2}}{m_{\phi, h}^{2}}\right)} \\
& \times \sqrt{\left(1-\frac{m_{\phi, h}^{2}}{m_{\chi}^{2}}\right)\left(1-\frac{4 m_{j}^{2}}{m_{\phi, h}^{2}}\right)-\left(1-\frac{2 E_{j}}{m_{\chi}}\right)^{2}} .
\end{aligned}
$$

Finally, the gamma-ray spectrum in the center-of-mass frame of the annihilating DM can be written as

$$
\begin{aligned}
\left(\frac{d N_{j}}{d E_{\gamma}}\right)_{\phi \phi}= & \int_{E_{\min }^{\phi}}^{E_{\max }^{\phi}} d E_{j} \frac{a_{j} E_{j}^{1 / 2}}{E_{\gamma}^{3 / 2}} e^{-b_{j} E_{\gamma} / E_{j}} 2\left(\frac{d P}{d E_{j}}\right)_{\phi}, \\
\left(\frac{d N_{j}}{d E_{\gamma}}\right)_{h h}= & \int_{E_{\min }^{h}}^{E_{\max }^{h}} d E_{j} \frac{a_{j} E_{j}^{1 / 2}}{E_{\gamma}^{3 / 2}} e^{-b_{j} E_{\gamma} / E_{j} 2}\left(\frac{d P}{d E_{j}}\right)_{h}, \\
\left(\frac{d N_{j}}{d E_{\gamma}}\right)_{\phi h}= & \int_{E_{\min }^{\phi}}^{E_{\max }^{\phi}} d E_{j} \frac{a_{j} E_{j}^{1 / 2}}{E_{\gamma}^{3 / 2}} e^{-b_{j} E_{\gamma} / E_{j}} \\
& \times\left(\left(\frac{d P}{d E_{j}}\right)_{\phi}+\left(\frac{d P}{d E_{j}}\right)_{h}\right),
\end{aligned}
$$

where $\left(a_{j}, b_{j}\right)$ are power-law parameters, the values of which can be obtained from the simulation [20,21]. The values of the parameters are shown to be

$$
\left(a_{j}, b_{j}\right)=(1.0,10.7),(1.1,15.1),(0.95,6.5),(0.73,7.76)
$$

for bottom quarks, top quarks, up quarks, and gauge bosons final states.

In the case of light mediator $m_{\phi}<m_{j}$ and $m_{\phi}<2 m_{h}$, where $\phi$ cannot decay into particles outside the SM, the branching ratios of $\phi$ is similar to those of the Higgs boson since $\phi$ decays through its mixing with the Higgs boson. In a general case, the $\phi$ branching ratios can be determined from its partial decay widths given in Appendix B. Although sterile neutrinos are unstable and subsequently decay into SM particles via off-shell $\phi$, such decay involves three-particle final states, at least one of which is a neutrino, $\phi \rightarrow \nu+$ SM + SM. Similarly, the two Higgs final states subsequently decay into multiple SM particles. The electromagnetic showering energy from these channels is therefore assumed to be subdominant and irrelevant to our study. We will further approximate that for each mass range of the scalar particle the decay is $100 \%$ into the largest contribution to the decay width. Therefore, the power spectrum is chosen according to the final states as

$$
(a, b)= \begin{cases}(0.73,7.76), & 160 \mathrm{GeV} \leq m_{\phi}, \\ (1.0,10.7), & 125 \mathrm{GeV}<m_{\phi}<160 \mathrm{GeV} .\end{cases}
$$

For an illustrative example, let us investigate the gammaray flux from DM annihilation in Eq. (32). For simplicity, we consider then extreme case in which DM annihilates 

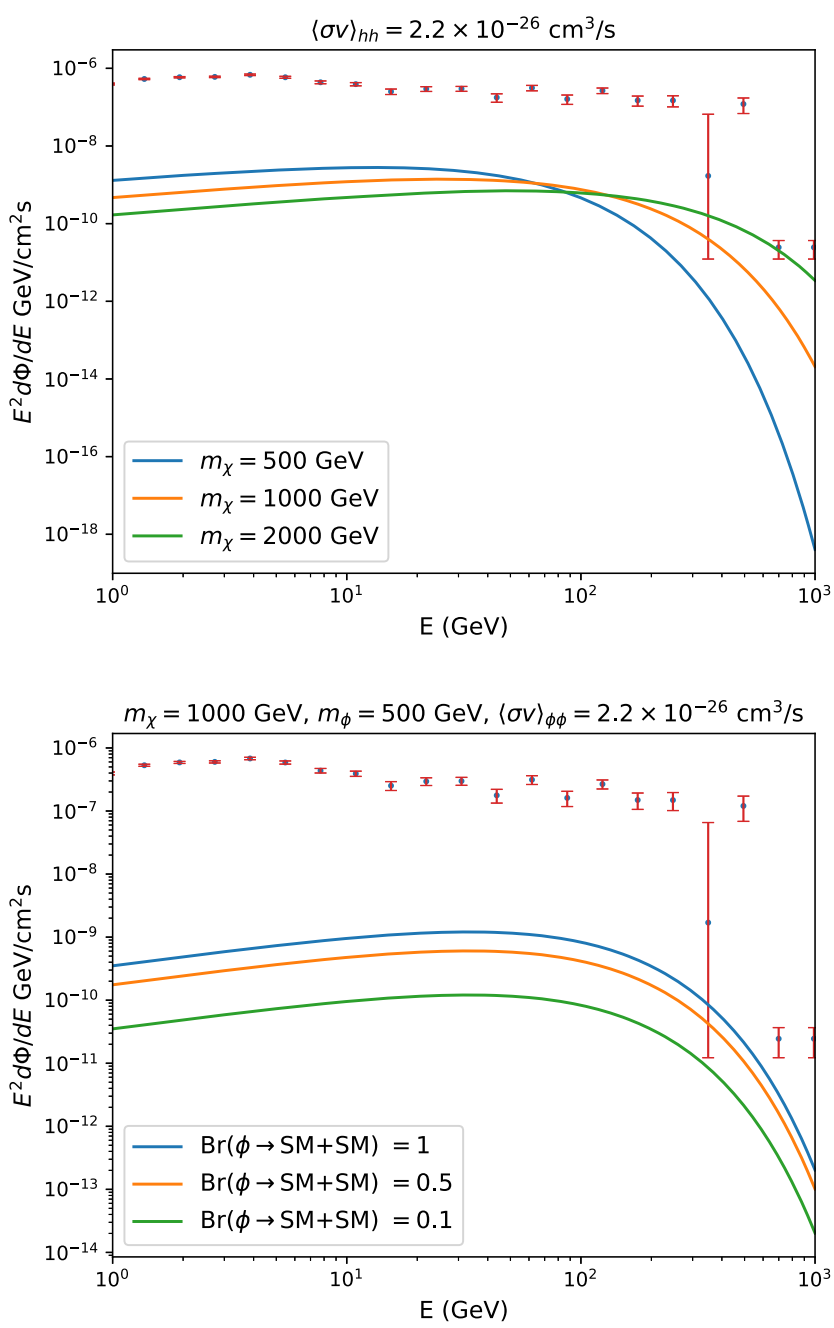

FIG. 2. Gamma-ray flux from DM annihilation into $h h$ (above) and $\phi \phi$ (below).

through a single channel and its annihilation cross section is equal to the thermal relic value. In the case in which DM annihilates into a pair of $h$, the gamma-ray flux is shown in the top pane of Fig. 2. The bottom panel shows the gammaray flux in the case in which DM annihilates into $\phi \phi$ with $m_{\phi}=500 \mathrm{GeV}$. We see that for these two extreme cases the fluxes differ by less than an order of magnitude. However, in computing the gamma-ray flux for a particular model parameter point, collectively represented by $\left(\langle\sigma v\rangle_{\text {scalar }}, m_{\chi}, m_{\phi}\right)$, one needs to take into account annihilation through all three channels in Eq. (32). We perform such a computation in Sec. IV.

Let us end this subsection with a remark regarding the Fermi excess. Because of the high tail behavior of the excess from the Fermi telescope, the flux from DM annihilation cannot possibly explain all bins of the excess. It is possible that some part of the energy range might originate from other astrophysical sources. In this project, we will use the excess as the upper limit on the modelgenerating flux.

\section{Neutrino telescope}

The neutrino produced from DM annihilation carries a definite energy depending on the decay channel,

$$
E_{\nu}=\left\{\begin{array}{ll}
m_{\chi} & \chi \bar{\chi} \rightarrow \nu \nu \\
\frac{4 m_{\chi}^{2}-m_{\psi}^{2}}{4 m_{\chi}} & \chi \bar{\chi} \rightarrow \nu \psi_{2}, \nu \psi_{3}
\end{array},\right.
$$

where we have used $\nu \equiv \psi_{1}$ for the lightest neutrino. The flux is therefore written as

$$
\begin{aligned}
\frac{d \Phi}{d E_{\nu}}= & \frac{1}{3} \frac{J R_{\mathrm{sc}} \rho_{\mathrm{sc}}^{2}}{8 \pi m_{\chi}^{2}}\left[\langle\sigma v\rangle_{\nu \nu} \delta\left(E_{\nu}-m_{\chi}\right)\right. \\
& \left.+\sum_{i=2}^{3}\langle\sigma v\rangle_{\nu \psi_{i}} \delta\left(E_{\nu}-\frac{4 m_{\chi}^{2}-m_{\psi_{i}}^{2}}{4 m_{\chi}}\right)\right],
\end{aligned}
$$

where we have focused on the electron-neutrino flux only. The 1/3 factor in the above equation arises from the assumption that the flavor ratio of neutrinos arriving at Earth is $\nu_{e}: \nu_{\mu}: \nu_{\tau} \simeq 1: 1: 1$.

In our analysis, we have reinterpreted the IceCUBE data for the DM search in our scenario. It turns out that the annihilation cross section into neutrinos from our model is less than $10^{-31} \mathrm{~cm}^{3} / \mathrm{s}$, which is well below the recast IceCUBE limit (approximately $10^{-24} \mathrm{~cm}^{3} / \mathrm{s}$ ).

\section{Direct detection}

In the direct detection experiment, the momentum exchanged between the DM and nucleon is typically much smaller than the mass of the scalar mediator. Thus, it is convenient to describe DM-nucleon interaction with the effective operator. In our model, the effective operator for DM-nucleon interaction reads

$$
\mathcal{L}_{\chi N}=s_{2 \theta}\left(\frac{1}{m_{\phi}^{2}}-\frac{1}{m_{h}^{2}}\right) \bar{\chi}\left(G+i \tilde{G} \gamma_{5}\right) \chi \mathcal{S}_{q}
$$

where $\mathcal{S}_{q}$ is the scalar current representing the interaction between the mediator and the quarks inside the nucleon. In the case in which the momentum exchanged is smaller than the heavy quarks, the scalar current is given by

$$
\mathcal{S}_{q}=\sum_{q=u, d, s} \frac{m_{q}}{v} \bar{q} q-\frac{\alpha_{s}}{4 \pi v} G_{\mu \nu}^{a} G^{a \mu \nu}
$$

where the gluonic term arises from integrating out the heavy quarks. In the case of heavy DM $\left(m_{\chi} \geq 1 \mathrm{TeV}\right)$, the momentum exchanged can be comparable to the charm mass. For such a case, one needs to take into account the charm mass threshold effect. However, we will ignore the charm mass effect in the rest of this work. 
The amplitude for DM-nucleon scattering depends on the $\mathcal{S}_{q}$ nucleon matrix elements. They are conventionally parametized in terms of the quark and the gluonic form factors [22]

$$
\begin{aligned}
\left\langle N\left|m_{q} \bar{q} q\right| N\right\rangle & =m_{N} f_{T q}^{(N)}, \\
\left\langle N\left|\frac{\alpha_{s}}{4 \pi} G_{\mu \nu}^{a} G^{a \mu \nu}\right| N\right\rangle & =-\frac{2}{9} m_{N} f_{T G}^{(N)} .
\end{aligned}
$$

The gluonic form factor is related to the quark form factors by the QCD trace anomaly in the heavy quark limit [23]

$$
f_{T G}^{(N)}=1-\sum_{q=u, d, s} f_{T q}^{(N)} .
$$

Thus, the nucleon matrix element of the scalar current is

$$
f_{N} \equiv\left\langle N\left|S_{q}\right| N\right\rangle=\frac{2}{9} \frac{m_{N}}{v}\left(1+\frac{7}{2} \sum_{q=u, d, s} f_{T q}^{(N)}\right) .
$$

For numerical analysis, we take the strange quark form factor to be $f_{T s}^{(p)}=f_{T s}^{(n)}=0.043 \pm 0.011$ [24]. We extract the up and the down quark form factors from the pion-nucleon sigma term, $\sigma_{\pi N}$, using the relations provided by Ref. [25]. However, there is a discrepancy between the values of $\sigma_{\pi N}$ extracted from the scattering data using baryon chiral effective theory and the lattice computation. We follow Ref. [26] and conservatively take $\sigma_{\pi N}=50 \pm 15 \mathrm{MeV}$. Thus, we determine the $u$ and $d$ quark form factors to be

$f_{T u}^{(p)}=(1.8 \pm 0.5) \times 10^{-2}, \quad f_{T u}^{(n)}=(1.6 \pm 0.5) \times 10^{-2}$, $f_{\text {Td }}^{(p)}=(3.4 \pm 1.1) \times 10^{-2}, \quad f_{\text {Td }}^{(n)}=(3.8 \pm 1.1) \times 10^{-2}$.

Therefore, to a good approximation, the nucleon matrix elements, Eq. (40), for the proton and the neutron are the same $\langle p|S| p\rangle \simeq\langle n|S| n\rangle$.

Armed with the DM-nucleon matrix element, we determine the spin-average DM-nucleon scattering amplitude squared, in the zero momentum transferred limit, to be

$$
|\mathcal{M}|^{2}=4 f_{N}^{2}\left(G^{2}+\tilde{G}^{2}\right) s_{2 \theta}^{2} m_{N}^{2} m_{\chi}^{2}\left(\frac{1}{m_{\phi}^{2}}-\frac{1}{m_{h}^{2}}\right)^{2} .
$$

Finally, we determine the DM-nucleon scattering cross section to be

$\sigma_{\chi N}=\frac{f_{N}^{2}\left(G^{2}+\tilde{G}^{2}\right) s_{2 \theta}^{2}}{4 \pi} \frac{m_{\chi}^{2} m_{N}^{2}}{\left(m_{\chi}+m_{N}\right)^{2}}\left(\frac{1}{m_{\phi}^{2}}-\frac{1}{m_{h}^{2}}\right)^{2}$.

The current upper limit is reported by the XENON1T Collaboration [27].

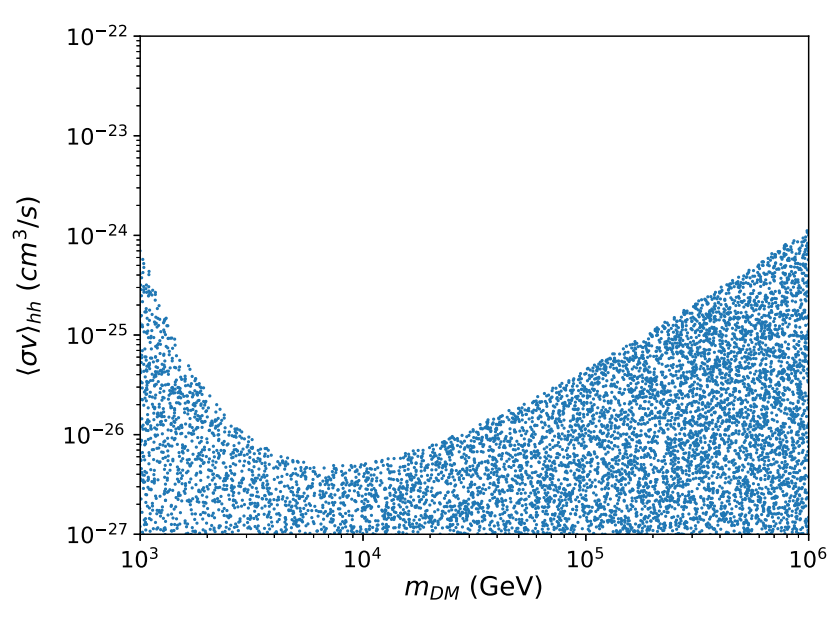

FIG. 3. Cross section for DM annihilation into a pair of Higgs bosons consistent with constraints from the light neutrino mass limit, the invisible $Z$ decay width, the $125 \mathrm{GeV}$ Higgs data, and the Fermi gamma-ray excess.

\section{RESULTS}

We approach the phenomenology of the model by scanning parameter space subjected to all constraints. We first consider the mass scale from the following random sets:

$M, m_{\phi}, v_{\phi} \in\left[10^{2}, 10^{6}\right] \mathrm{GeV}, \quad|\cos \theta| \in[0.9931,1]$.

Then, we calculate the couplings in the scalar sector using Eq. (21). Demanding that all couplings are perturbative, we apply the constraints on $\lambda_{\phi}, \lambda_{h}, \lambda_{\phi h}<4 \pi$. The next step in generating a set of parameters is to consider the neutrino sector. The set of parameters $(y, g)$ is generated from the following range:

$$
y, g \in\left[10^{-3}, \sqrt{4 \pi}\right] .
$$

The constraint on the $Z$ invisible decay width from Eq. (27) is then applied to the parameter set. Finally, the rest of parameters are chosen as follows:

$$
G, \tilde{G} \in\left[10^{-3}, \sqrt{4 \pi}\right], \quad \mu_{N} \in\left[10^{-10}, 10^{-7}\right] \mathrm{GeV} .
$$

After we obtain the complete set of model parameter space, the mass spectrum of the theory is then calculated from Eqs. (8), (18), and (23). The DM annihilation cross sections are computed using the expression given in Appendix A. Then, the neutrino mass limit is applied $\left(m_{\nu}<0.2 \mathrm{eV}\right)$. Next, we use Eq. (29) to produce gammaray flux for each point of the set. Then, we impose the excess reported by Fermi-LAT Collaboration as the upper limit for the flux from DM annihilation [19]. Note that from Fig. 3 the gamma-ray constraint has a clear impact on the 


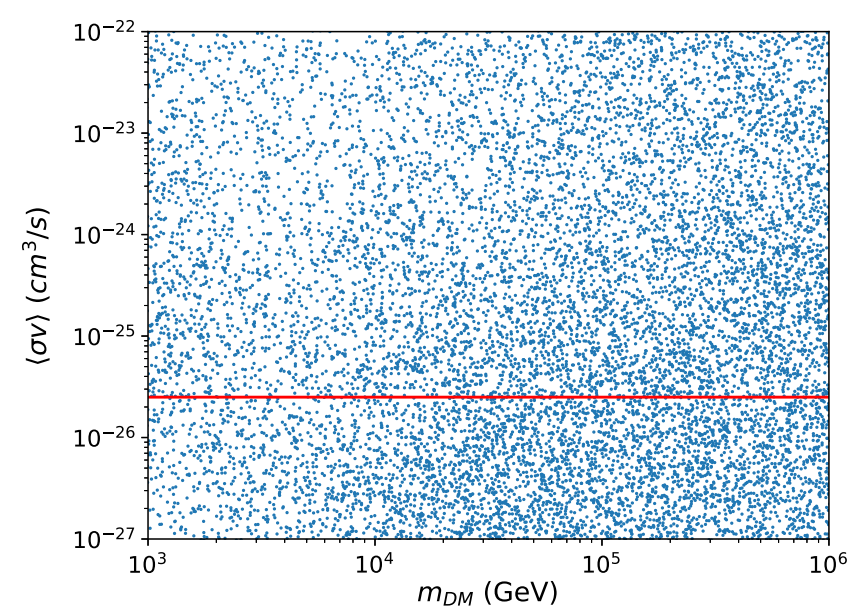

FIG. 4. Total DM annihilation cross section consistent with constraints from the light neutrino mass limit, the invisible $Z$ decay width, the $125 \mathrm{GeV}$ Higgs data, and the Fermi gamma-ray excess. The red line is the thermal relic annihilation cross section.

annihilation cross section into two Higgs final states as expected since the branching ratio of the scalar mediator to the SM particles is often found to be too small.

The total DM annihilation cross section of the remaining parameter points are shown in Fig. 4, in which the thermal relic density $\langle\sigma v\rangle_{\text {total }}=2.5 \times 10^{-26} \mathrm{~cm}^{3} / \mathrm{s}$ is shown as the red line. To prevent the Universe from being overclosed, we impose the constraint $\langle\sigma v\rangle_{\text {total }}>$ $2.5 \times 10^{-26} \mathrm{~cm}^{3} / \mathrm{s}$. Finally, we calculate the DM-nucleon scattering cross section on the remaining parameter points using Eq. (43). In Fig. 5, the result is shown together with the upper limit reported by the XENON1T Collaboration.

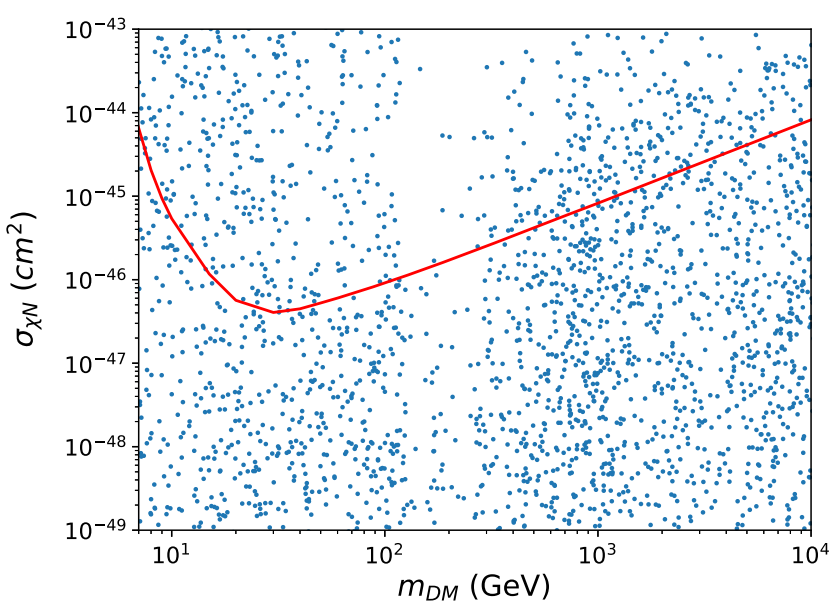

FIG. 5. DM-nucleon scattering cross section consistent with constraints from the light neutrino mass limit, the invisible $Z$ decay width, the $125 \mathrm{GeV}$ Higgs data, the Fermi gamma-ray excess, and freeze-out production. The red line is the upper bound from XENON1T.

\section{SUMMARY AND OUTLOOK}

In this work, we consider a class of models in which the neutrino mass can be explained by the inverse seesaw mechanism. The smallness of the active neutrino mass is achieved through the dynamical Majorana mass of two additional sterile neutrinos. The extra scalar field is connected to the DM sector, giving a strong connection between the neutrino and DM.

We have identified the viable parameter space of the model consistent with constraints from the light neutrino mass limit, the invisible $Z$ decay width, the $125 \mathrm{GeV}$ Higgs measurements, and the Fermi gamma-ray excess. We find that the Fermi gamma-ray excess places a strong constraint on the DM annihilation to a pair of Higgs bosons. However, a large chunk of parameter space still remains open, as can be seen in Fig. 5. These parts of parameter space could be probed by the next generation of direct and indirect detection experiments. It is interesting to study the sensitivities of the upcoming direct detection experiments such as LZ and XENONnT as well as indirect detection experiment such as the Cherenkov Telescope Array on this model. We leave such study for possible future work.

Finally, we want to remark that our model is not yet fully realistic in the sense that it only contains one massive light neutrino. However, one can easily extend the model by introducing an additional pair of sterile neutrinos and a flavor symmetry. We do not expect it to have a significant impact on DM phenomenology, which is the main goal of the paper.

\section{ACKNOWLEDGMENTS}

The work of C.P. and P. U. has been supported in part by the Thailand Research Fund under Contracts No. MRG6280131 and No. MRG6280186 and by the National Astronomical Research Institute of Thailand. P. U. also acknowledges the support of the Faculty of Science, Srinakharinwirot University. N. T. is supported by the scholarship from the Development and Promotion of Science and Technology Talents Project. We thank the referee for his/her careful reading of our paper and for the valuable comments and suggestions that helped improve our paper.

\section{APPENDIX A: ANNIHILATION CROSS SECTION}

The DM annihilation cross section into a pair of SM fermions and gauge bosons is

$$
\begin{aligned}
\langle\sigma v\rangle_{W W}= & \frac{\left(G^{2} \beta_{\chi}^{2}+\tilde{G}^{2}\right) s_{2 \theta}^{2}}{64 \pi v^{2}}\left(1-r_{W}+\frac{3}{4} r_{W}^{2}\right) \\
& \times \beta_{W}\left(\frac{s}{s-m_{h}^{2}}-\frac{s}{s-m_{\phi}^{2}}\right)^{2},
\end{aligned}
$$




$$
\begin{aligned}
\langle\sigma v\rangle_{Z Z}= & \frac{\left(G^{2} \beta_{\chi}^{2}+\tilde{G}^{2}\right) s_{2 \theta}^{2}}{128 \pi v^{2}}\left(1-r_{Z}+\frac{3}{4} r_{Z}^{2}\right) \\
& \times \beta_{Z}\left(\frac{s}{s-m_{h}^{2}}-\frac{s}{s-m_{\phi}^{2}}\right)^{2}, \\
\langle\sigma v\rangle_{\bar{\psi} \psi}= & \frac{\left(G^{2} \beta_{\chi}^{2}+\tilde{G}^{2}\right) s_{2 \theta}^{2}}{32 \pi} \frac{m_{\psi}^{2} s \beta_{\psi}^{3}}{v^{2}} \\
& \times\left(\frac{1}{s-m_{h}^{2}}-\frac{1}{s-m_{\phi}^{2}}\right)^{2},
\end{aligned}
$$

where $r_{x}=m_{x}^{2} / s$ and $\beta_{\chi}=\sqrt{1-\frac{4 m_{\chi}^{2}}{s}}$. The cross section for DM annihilating into a pair of scalar bosons is given by

$$
\begin{aligned}
\langle\sigma v\rangle_{h h}= & \frac{\beta_{\chi}^{2} G^{2}+\tilde{G}^{2}}{32 \pi} \sqrt{1-\frac{m_{h}^{2}}{m_{\chi}^{2}}}\left(\frac{4 m_{\chi} G s_{\theta}^{2}}{2 m_{\chi}^{2}-m_{h}^{2}}\right. \\
& \left.-\frac{s_{\theta} \lambda_{h h h}}{4 m_{\chi}^{2}-m_{h}^{2}}-\frac{c_{\theta} \lambda_{\phi h h}}{4 m_{\chi}^{2}-m_{\phi}^{2}}\right)^{2}, \\
\langle\sigma v\rangle_{\phi \phi}= & \frac{\beta_{\chi}^{2} G^{2}+\tilde{G}^{2}}{32 \pi} \sqrt{1-\frac{m_{\phi}^{2}}{m_{\chi}^{2}}\left(\frac{4 m_{\chi} G s_{\theta}^{2}}{2 m_{\chi}^{2}-m_{\phi}^{2}}\right.} \\
& \left.-\frac{s_{\theta} \lambda_{\phi h h}}{4 m_{\chi}^{2}-m_{h}^{2}}-\frac{c_{\theta} \lambda_{h h h}}{4 m_{\chi}^{2}-m_{\phi}^{2}}\right)^{2}, \\
\langle\sigma v\rangle_{h \phi}= & \frac{\beta_{\chi}^{2} G^{2}+\tilde{G}^{2}}{16 \pi m_{\chi}^{2}} \sqrt{\lambda\left[\frac{m_{h}^{2}}{4 m_{\chi}^{2}}, \frac{m_{\phi}^{2}}{4 m_{\chi}^{2}}\right]} \\
\times & \left(\frac{8 m_{\chi} G s_{\theta} c_{\theta}}{4 m_{\chi}^{2}-m_{h}^{2}-m_{\phi}^{2}}-\frac{s_{\theta} \lambda_{\phi h h}}{4 m_{\chi}^{2}-m_{h}^{2}}-\frac{c_{\theta} \lambda_{h \phi \phi}}{4 m_{\chi}^{2}-m_{\phi}^{2}}\right)^{2},
\end{aligned}
$$

where $\lambda(x, y)=1+x^{2}+y^{2}-2 x-2 y-2 x y$ is the phase space factor and

$$
\begin{aligned}
\lambda_{h h h}= & -\frac{6 s_{\theta}^{3}\left(m_{\phi}^{2} c_{\theta}^{2}+m_{h}^{2} s_{\theta}^{2}\right)}{v_{\phi}}-\frac{6 c_{\theta}^{3}\left(m_{h}^{2} c_{\theta}^{2}+m_{\phi}^{2} s_{\theta}^{2}\right)}{v} \\
& -\frac{\left(m_{\phi}^{2}-m_{h}^{2}\right) c_{\theta} s_{\theta}}{v v_{\phi}}\left(3 v_{\phi} c_{\theta}^{2} s_{\theta}+3 v c_{\theta} s_{\theta}^{2}\right), \\
\lambda_{\phi h h}= & -3 s_{2 \theta}\left[m_{\phi}^{2}\left(\frac{c_{\theta} s_{\theta}^{2}}{v_{\phi}}+\frac{c_{\theta}^{2} s_{\theta}}{v}\right)+m_{h}^{2}\left(\frac{c_{\theta}^{3}}{v_{\phi}}+\frac{s_{\theta}^{3}}{v}\right)\right] \\
& -\frac{\left(m_{\phi}^{2}-m_{h}^{2}\right) c_{\theta} s_{\theta}}{v v_{\phi}}\left(v_{\phi} c_{\theta}^{3}+2 v c_{\theta}^{2} s_{\theta}-2 v_{\phi} c_{\theta} s_{\theta}^{2}-v s_{\theta}^{3}\right),
\end{aligned}
$$

$$
\begin{aligned}
\lambda_{h \phi \phi}= & -3 s_{2 \theta}\left[m_{\phi}^{2}\left(\frac{c_{\theta}^{3}}{v_{\phi}}+\frac{s_{\theta}^{3}}{v}\right)+m_{h}^{2}\left(\frac{c_{\theta} s_{\theta}^{2}}{v_{\phi}}+\frac{c_{\theta}^{2} s_{\theta}}{v}\right)\right] \\
& -\frac{\left(m_{\phi}^{2}-m_{h}^{2}\right) c_{\theta} s_{\theta}}{v v_{\phi}}\left(v_{\phi} s_{\theta}^{3}-2 v c_{\theta} s_{\theta}^{2}-2 v_{\phi} c_{\theta}^{2} s_{\theta}+v c_{\theta}^{3}\right) \\
\lambda_{\phi \phi \phi}= & -\frac{6 c_{\theta}^{3}\left(m_{\phi}^{2} c_{\theta}^{2}+m_{h}^{2} s_{\theta}^{2}\right)}{v_{\phi}}+\frac{6 s_{\theta}^{3}\left(m_{h}^{2} c_{\theta}^{2}+m_{\phi}^{2} s_{\theta}^{2}\right)}{v} \\
& -\frac{\left(m_{\phi}^{2}-m_{h}^{2}\right) c_{\theta} s_{\theta}}{v v_{\phi}}\left(3 v_{\phi} c_{\theta} s_{\theta}^{2}-3 v c_{\theta}^{2} s_{\theta}\right)
\end{aligned}
$$

Finally, the annihilation cross sections into neutrinos are given by

$$
\begin{aligned}
\langle\sigma v\rangle_{\psi_{i} \psi_{j}}= & \frac{\beta_{\chi}^{2} G^{2}+\tilde{G}^{2}}{16 \pi}\left(\frac{s_{\theta} y_{h i j}}{s-M_{h}^{2}}+\frac{c_{\theta} y_{\phi i j}}{s-M_{\phi}^{2}}\right)^{2} \\
& \times \sqrt{s-\left(m_{i}+m_{j}\right)^{2}} \sqrt{\lambda\left[\frac{m_{i}^{2}}{s}, \frac{m_{j}^{2}}{s}\right]},
\end{aligned}
$$

where

$$
\begin{gathered}
y_{h i i}=\frac{y}{\sqrt{2}} c_{\theta} R_{1 i} R_{2 i}+g s_{\theta} R_{2 i} R_{3 i}, \\
y_{\phi i i}=\frac{-y}{\sqrt{2}} s_{\theta} R_{1 i} R_{2 i}+g c_{\theta} R_{2 i} R_{3 i}, \\
y_{h i j}=-\frac{1}{\sqrt{2}} y c_{\theta}\left(R_{1 i} R_{2 j}+R_{1 j} R_{2 i}\right)-g s_{\theta}\left(R_{2 i} R_{3 j}+R_{2 j} R_{3 i}\right),
\end{gathered}
$$

$y_{\phi i j}=+\frac{1}{\sqrt{2}} y s_{\theta}\left(R_{1 i} R_{2 j}+R_{1 j} R_{2 i}\right)-g c_{\theta}\left(R_{2 i} R_{3 j}+R_{2 j} R_{3 i}\right)$.

\section{APPENDIX B: DECAY WIDTH}

We discuss the decay width of the scalar mediator in this section. Because of mixing in the scalar sector, the decay width of $\phi$ into the SM particles takes the form

$$
\begin{gathered}
\Gamma(\phi \rightarrow V V)=\frac{\sin ^{2} \theta}{32 \pi} \frac{m_{\phi}^{3}}{v^{2}} \delta_{V} \sqrt{1-4 x}\left(1-4 x+12 x^{2}\right), \\
\Gamma(\phi \rightarrow \bar{f} f)=\frac{N_{c} \sin ^{2} \theta}{8 \pi} \frac{m_{\phi} m_{f}^{2}}{v^{2}} \beta_{f}^{3},
\end{gathered}
$$


where $V=W, Z, \delta_{W}=2, \delta_{Z}=1, x=m_{V}^{2} / m_{\phi}^{2}$, and $\beta_{f}=\sqrt{1-\frac{4 m_{f}^{2}}{m_{\phi}^{2}}}$. The decay width to neutrinos can be obtained straightforwardly:

$\Gamma\left(\phi \rightarrow \psi_{i} \psi_{j}\right)=\frac{1}{32 \pi} y_{\phi i j}^{2} \sqrt{m_{\phi}^{2}-\left(m_{i}-m_{j}\right)^{2}} \lambda\left[\frac{m_{i}^{2}}{s}, \frac{m_{j}^{2}}{s}\right]^{3 / 2}$.
Finally, for a sufficiently heavy $\phi$, it can decay into two Higgs bosons,

$$
\Gamma(\phi \rightarrow h h)=\frac{1}{32 \pi} \frac{\lambda_{\phi h h}^{2}}{m_{\phi}}\left(1-\frac{4 m_{h}^{2}}{m_{\phi}^{2}}\right)^{1 / 2} .
$$

[1] R. N. Mohapatra and J. W. F. Valle, Phys. Rev. D 34, 1642 (1986).

[2] W.-C. Huang and F. F. Deppisch, Phys. Rev. D 91, 093011 (2015).

[3] W.-F. Chang and J. N. Ng, Phys. Rev. D 90, 065034 (2014).

[4] M. Escudero, N. Rius, and V. Sanz, J. High Energy Phys. 02 (2017) 045.

[5] M. Escudero, N. Rius, and V. Sanz, Eur. Phys. J. C 77, 397 (2017).

[6] V. De Romeri, E. Fernandez-Martinez, J. Gehrlein, P. A. N. Machado, and V. Niro, J. High Energy Phys. 10 (2017) 169.

[7] H. Ishida, K. S. Jeong, and F. Takahashi, Phys. Lett. B 731, 242 (2014).

[8] B. W. Lee and R. E. Shrock, Phys. Rev. D 16, 1444 (1977).

[9] W. J. Marciano and A. I. Sanda, Phys. Lett. 67B, 303 (1977).

[10] S. T. Petcov, Yad. Fiz. 25, 1336(E) (1977) [Sov. J. Nucl. Phys. 25, 340 (1977)].

[11] P. B. Pal and L. Wolfenstein, Phys. Rev. D 25, 766 (1982).

[12] R. Primulando and P. Uttayarat, J. High Energy Phys. 06 (2018) 026.

[13] A. Ahriche, S. M. Boucenna, and S. Nasri, Phys. Rev. D 93, 075036 (2016).

[14] G. Aad et al. (ATLAS and CMS Collaborations), J. High Energy Phys. 08 (2016) 045.
[15] M. Aaboud et al. (ATLAS Collaboration), Phys. Rev. D 98, 052005 (2018).

[16] A. M. Sirunyan et al. (CMS Collaboration), Eur. Phys. J. C 79, 421 (2019).

[17] M. Tanabashi et al. (Particle Data Group), Phys. Rev. D 98, 030001 (2018).

[18] M. G. Aartsen et al. (IceCube Collaboration), Eur. Phys. J. C 77, 627 (2017).

[19] M. Ackermann et al. (Fermi-LAT Collaboration), Astrophys. J. 840, 43 (2017).

[20] L. Bergstrom, P. Ullio, and J. H. Buckley, Astropart. Phys. 9, 137 (1998).

[21] J. L. Feng, K. T. Matchev, and F. Wilczek, Phys. Rev. D 63, 045024 (2001).

[22] G. Jungman, M. Kamionkowski, and K. Griest, Phys. Rep. 267, 195 (1996).

[23] M. A. Shifman, A. I. Vainshtein, and V. I. Zakharov, Phys. Lett. 78B, 443 (1978).

[24] P. Junnarkar and A. Walker-Loud, Phys. Rev. D 87, 114510 (2013).

[25] A. Crivellin, M. Hoferichter, and M. Procura, Phys. Rev. D 89, 054021 (2014).

[26] F. Bishara, J. Brod, P. Uttayarat, and J. Zupan, J. High Energy Phys. 01 (2016) 010.

[27] E. Aprile et al. (XENON Collaboration), Phys. Rev. Lett. 119, 181301 (2017). 